\title{
Quantum effects in anti-de Sitter spacetime for the electromagnetic vacuum
}

\section{A. S. Kotanjyan*}

Department of Physics, Yerevan State University, 1 Alex Manoogian Street, 0025 Yerevan, Armenia

E-mail: anna.kotanjyaneysu.am

\author{
A. A. Saharian \\ Department of Physics, Yerevan State University, 1 Alex Manoogian Street, 0025 Yerevan, \\ Armenia \\ E-mail: saharian@ysu.am

\section{H. G. Sargsyan} \\ Department of Physics, Yerevan State University, 1 Alex Manoogian Street, 0025 Yerevan, \\ Armenia \\ E-mail: hayk28.sargsyan@yahoo.com

\section{H. Simonyan} \\ Department of Physics, Yerevan State University, 1 Alex Manoogian Street, 0025 Yerevan, \\ Armenia \\ E-mail: david.simonyan94@mail.ru
}

\begin{abstract}
The two-point functions of the vector potential and of the field tensor for the electromagnetic field in background of anti-de Sitter (AdS) spacetime are evaluated. First we consider the twopoint functions in the boundary-free geometry and then generalize the results in the presence of a reflecting boundary parallel to the AdS horizon. By using the expressions for the two-point functions of the field tensor, we investigate the vacuum expectation values of the electric field squared and of the energy-momentum tensor. Simple asymptotic expressions are provided near the AdS boundary and horizon.
\end{abstract}

The Modern Physics of Compact Stars 2015

30 September 2015 - 3 October 2015

Yerevan, Armenia

\footnotetext{
*Speaker.
} 


\section{Introduction}

The prediction of nontrivial properties of the vacuum is among the most interesting results of quantum field theory. These properties are manifested in the response of the vacuum under external influences. In particular, it is of interest to consider the influence of the classical gravitational field on the characteristics of quantum vacuum. The corresponding problems are exactly solvable for highly symmetric background geometries only. In particular, the investigations of quantum effects in anti-de Sitter (AdS) spacetime have attracted a great deal of attention. There are several reasons for that. Much of early interest to AdS spacetime was motivated by the questions of principle nature related to the quantization of fields propagating on curved backgrounds. The lack of global hyperbolicity and the presence of both regular and irregular modes give rise to a number of new features which have no analogues in quantum field theory on the Minkowski bulk. The importance of this theoretical work increased when it was realized that AdS spacetime emerges as a stable ground state solution in extended supergravity and Kaluza-Klein models and in string theories. The appearance of the AdS/CFT correspondence and braneworld models of Randall-Sundrum type has revived interest in this subject considerably.

The boundary conditions, imposed on the field operator, modify the spectrum of the vacuum fluctuations of a quantum field. As a consequence, the vacuum expectation values (VEVs) of physical observables are shifted. This is the well known Casimir effect (for reviews see [1]). The investigations of the Casimir effect on AdS bulk have attracted a great deal of attention. The Casimir energy and the corresponding forces for two parallel branes in AdS spacetime have been evaluated in Refs. [2], both for scalar and fermionic fields, by using either dimensional or zeta function regularization methods. Local Casimir densities were considered in Refs. [3]. The Casimir effect in higher-dimensional generalizations of the AdS spacetime with compact internal spaces has been investigated in [4]. Induced vacuum currents for a charged scalar field in AdS background with toroidally compactified spatial dimensions have been recently studied in [5].

In the present paper we consider the influence of the plate parallel to the AdS boundary on the properties of the electromagnetic field. On the plate the boundary condition is imposed that is a generalization of the perfect conductor boundary condition in 4-dimensional spacetime. The two-point function for the electromagnetic field in the boundary-free AdS spacetime is investigated in [6]. The electromagnetic Casimir densities in de Sitter spacetime for flat boundaries have been considered in [7]. The electromagnetic two-point functions and the Casimir effect in background of more general Friedmann-Robertson-Walker cosmologies are discussed in [8].

The outline of our investigation is the following. First we consider the mode functions for the electromagnetic field for the AdS background described in Poíncare coordinates. In Section 3 we consider the two-point functions for the vector potential and for the field tensor in AdS spacetime when the boundary is absent. The mode functions and the two-point functions in the geometry with a plate parallel to the AdS boundary are discussed in Section 4. Our main interest are the Casimir densities and, in Section 5, we study the VEV of the energy-momentum tensor for both the regions on the right and on the left of the boundary. 


\section{Electromagnetic field modes}

Consider the electromagnetic field in background of $(D+1)$-dimensional AdS spacetime. In the Poincaré coordinates the corresponding line element has the form

$$
d s^{2}=g_{i k} d x^{i} d x^{k}=e^{-2 y / \alpha} \eta_{\mu v} d x^{\mu} d x^{v}-d y^{2}
$$

where $\eta_{\mu v}=\operatorname{diag}(1,-1, \ldots .-1)$ is the metric tensor for $D$-dimensional Minkowski spacetime and $\alpha$ is the curvature radius. The latter is related to the Ricci scalar $R$ of the background spacetime and to the cosmological constant $\Lambda$ by the formulae $R=-D(D+1) / \alpha^{2}$ and $\Lambda=-D(D-1) \alpha^{-2} / 2$. Here and below $i, k=0,1, \ldots, D$, and $\mu, v=0,1, \ldots, D-1$. By the coordinate transformation $z=$ $\alpha e^{y / \alpha}, 0 \leqslant z<\infty$, the line element is presented in a conformally-flat form

$$
d s^{2}=(\alpha / z)^{2} \eta_{i k} d x^{i} d x^{k},
$$

with $x^{D}=z$. For the action of the electromagnetic fields one has

$$
S=-\frac{1}{16 \pi} \int d^{D+1} x \sqrt{|g|} F_{i k}(x) F^{i k}(x),
$$

where $F_{i k}(x)=\partial_{i} A_{k}(x)-\partial_{k} A_{i}(x)$ is the field tensor. In what follows it is convenient to fix the gauge by the conditions $A_{D}=0, \nabla_{i} A^{i}=0$. For the line element (2.2) the second condition is reduced to $\partial_{\mu} A^{\mu}=0$.

We make the Fourier transform with respect to the coordinates $x^{\mu}, \mu=0, \ldots, D-1$ :

$$
A_{\mu}(x)=\int d^{D} k A_{\mu}(z, k) e^{-i \eta_{v \alpha} k^{v} x^{\alpha}}
$$

From the gauge conditions it follows that $k_{\mu} A^{\mu}(z, k)=0, k_{\mu}=\eta_{\mu \nu} k^{v}$. In terms of the Fourier components for the vector potential, the action is written in the form

$$
S=\frac{(2 \pi)^{D-1}}{4} \int d z \int d^{D} k(\alpha / z)^{D-3} \eta^{\mu v}\left[\partial_{D} A_{\mu}(z, k) \partial_{D} A_{v}^{*}(z, k)-\lambda^{2} A_{\mu}(z, k) A_{v}^{*}(z, k)\right],
$$

where we have introduced the notation $\lambda^{2}=k_{0}^{2}-k_{1}^{2}-\cdots-k_{D-1}^{2}$, with $k_{0}=\omega$ and the star stands for the complex conjugate.

For the variation of the action (2.5) with respect to $A_{v}^{*}(z, k)$ one gets

$$
\delta S=-\frac{(2 \pi)^{D-1}}{4} \int d z \int d^{D} k \eta^{\mu v}\left\{\partial_{D}\left[(\alpha / z)^{D-3} \partial_{D} A_{\mu}(z, k)\right]+(\alpha / z)^{D-3} \lambda^{2} A_{\mu}(z, k)\right\} \delta A_{v}^{*}(z, k),
$$

where we have assumed that on the limits of the integration over $z$ one has

$$
\left.(\alpha / z)^{D-3} \eta^{\mu v} \partial_{D} A_{\mu}(z, k) \delta A_{v}^{*}(z, k)\right|_{z}=0 .
$$

This limits depend on the problem at hand. In the boundary-free AdS spacetime they are given by $z=0$ and $z=\infty$. From $\delta S=0$ the following equation is obtained for the Fourier component:

$$
z^{D-3} \partial_{D}\left[z^{3-D} \partial_{D} A_{\mu}(z, k)\right]+\lambda^{2} A_{\mu}(z, k)=0 .
$$


The solution of this equation (2.8) is given by

$$
A_{\mu}(z, k)=\varepsilon_{(\sigma) \mu} z^{D / 2-1} Z_{D / 2-1}(\lambda z),
$$

where $Z_{v}(x)$ is a cylinder function of the order $v$ and $\varepsilon_{(\sigma) \mu}$ are polarization vectors. Here the index $\sigma$, with the values $\sigma=1, \ldots, D-1$, enumerates the $D-1$ independent polarizations for the electromagnetic field.

As a complete set of mode functions for the electromagnetic field one can take

$$
A_{(\sigma \lambda \mathbf{k}) \mu}=\varepsilon_{(\sigma) \mu} z^{D / 2-1} Z_{D / 2-1}(\lambda z) e^{i \mathbf{k} \cdot \mathbf{x}-i \omega t},
$$

where $\mathbf{x}=\left(x^{1}, x^{2}, \ldots, x^{D-1}\right)$ and $\mathbf{k}=\left(k^{1}, k^{2}, \ldots, k^{D-1}\right)$. We assume that the polarization vectors are normalized by the condition

$$
\eta^{\mu v} \varepsilon_{\left(\sigma^{\prime}\right) \mu} \varepsilon_{(\sigma) v}=-\delta_{\sigma \sigma^{\prime}}
$$

From the gauge conditions one has $\varepsilon_{(\sigma) D}=0$ and $k^{\mu} \varepsilon_{(\sigma) \mu}=0$. In the evaluation of the two-point functions we also need the sum $\sum_{\sigma=1}^{D-1} \varepsilon_{(\sigma) \mu} \varepsilon_{(\sigma) v}$. It can be seen that the following relation takes place:

$$
\sum_{\sigma=1}^{D-1} \varepsilon_{(\sigma) \mu} \varepsilon_{(\sigma) v}=-\eta_{\mu v}+\frac{k_{\mu} k_{v}}{\lambda^{2}} .
$$

The mode functions are normalized in accordance with the orthonormalization condition

$$
\int d^{D} x \sqrt{|g|}\left[A_{\left(\sigma^{\prime} \lambda^{\prime} \mathbf{k}^{\prime}\right) \mu}^{*} \nabla^{0} A_{(\sigma \lambda \mathbf{k})}^{\mu}-\left(\nabla^{0} A_{\left(\sigma^{\prime} \lambda^{\prime} \mathbf{k}^{\prime}\right) \mu}^{*}\right) A_{(\sigma \lambda \mathbf{k})}^{\mu}\right]=4 i \pi \delta_{\sigma \sigma^{\prime}} \delta_{\mathbf{k} \mathbf{k}^{\prime}} \delta_{\lambda \lambda^{\prime}} .
$$

By using the expression for the metric tensor we see that $A_{v}^{*} \nabla^{0} A^{v}-\left(\nabla^{0} A_{v}^{*}\right) A^{v}=-2 i \omega g^{00} A_{v}^{*} A^{v}$. Hence, the normalization condition takes the form

$$
\int d^{D} x \sqrt{|g|} g^{00} g^{\mu v} A_{\left(\sigma^{\prime} \lambda^{\prime} \mathbf{k}^{\prime}\right) \mu}^{*} A_{v(\sigma \lambda \mathbf{k})}=-\frac{2 \pi}{\omega} \delta_{\sigma \sigma^{\prime}} \delta_{\mathbf{k} \mathbf{k}^{\prime}} \delta_{\lambda \lambda^{\prime}}
$$

Substituting the mode functions (2.10) and by making use the relation (2.11) for the polarization vectors, for the function $Z_{D / 2-1}(\lambda z)$ we find

$$
\int d z z Z_{D / 2-1}\left(\lambda^{\prime} z\right) Z_{D / 2-1}(\lambda z)=\frac{\delta_{\lambda \lambda^{\prime}}}{(2 \pi)^{D-2} \alpha^{D-3} \omega} .
$$

The integration range depends on the problem under consideration. In the boundary-free geometry one has $0 \leqslant z<\infty$.

\section{Two-point functions in the boundary-free geometry}

Here, the only interaction of the quantum electromagnetic field is with the background gravitational field. As a result, all the information on the properties of the vacuum state are contained in the two-point functions. We will evaluate the two-point functions for the vector potential and for the field tensor. First we consider the case of the boundary-free AdS spacetime. For $D \geqslant 4$, from the normalizability condition of the modes on the AdS boundary it follows that in (2.10) we should take

$$
Z_{D / 2-1}(\lambda z)=C J_{D / 2-1}(\lambda z)
$$


where $J_{v}(x)$ is the Bessel function. Now, from the normalization condition (2.15), with the integration over the interval $(0, \infty)$, for the coefficient $C$ we get

$$
|C|^{2}=\frac{\lambda}{(2 \pi)^{D-2} \alpha^{D-3} \omega} .
$$

Hence, the electromagnetic field mode functions in the boundary-free geometry have the form

$$
A_{(\sigma \lambda \mathbf{k}) \mu}=C \varepsilon_{(\sigma) \mu} z^{D / 2-1} J_{D / 2-1}(\lambda z) e^{i \mathbf{k} \cdot \mathbf{x}-i \omega t} .
$$

The two-point function of the vector potential can be evaluated by using the mode-sum formula

$$
\left\langle 0\left|A_{\mu}(x) A_{v}\left(x^{\prime}\right)\right| 0\right\rangle \equiv\left\langle A_{\mu}(x) A_{v}\left(x^{\prime}\right)\right\rangle_{0}=\sum_{\sigma=1}^{D-1} \int d \mathbf{k} \int_{0}^{\infty} d \lambda A_{(\sigma \lambda \mathbf{k}) \mu}(x) A_{(\sigma \lambda \mathbf{k}) v}^{*}\left(x^{\prime}\right),
$$

where $|0\rangle$ stands for the vacuum state. Substituting the expressions for the mode functions, we get

$$
\begin{aligned}
\left\langle A_{\mu}(x) A_{v}\left(x^{\prime}\right)\right\rangle_{0}= & \frac{\left(z z^{\prime}\right)^{D / 2-1}}{(2 \pi)^{D-2} \alpha^{D-3}} \int d \mathbf{k} \int_{0}^{\infty} d \lambda \frac{\lambda}{\omega} e^{i \mathbf{k} \cdot \Delta \mathbf{x}-i \omega \Delta t} \\
& \times J_{D / 2-1}(\lambda z) J_{D / 2-1}\left(\lambda z^{\prime}\right)\left(-\eta_{\mu v}+\frac{k_{\mu} k_{v}}{\lambda^{2}}\right) .
\end{aligned}
$$

For the integral with the part containing the tensor $\eta_{\mu \nu}$ one has the following result

$$
\int d \mathbf{k} \int_{0}^{\infty} d \lambda \frac{\lambda}{\omega} J_{D / 2-1}(\lambda z) J_{D / 2-1}\left(\lambda z^{\prime}\right) e^{i \mathbf{k} \cdot \Delta \mathbf{x}-i \omega \Delta t}=\frac{2^{D-2} \pi^{(D-3) / 2} \Gamma((D-1) / 2)}{\left(z z^{\prime}\right)^{D / 2}\left(u^{2}-1\right)^{(D-1) / 2}}
$$

with the notation

$$
u=1+\left[(\Delta z)^{2}+|\Delta \mathbf{x}|^{2}-(\Delta t)^{2}\right] /\left(2 z z^{\prime}\right)
$$

The latter is related to the invariant distance between the points $x$ and $x^{\prime}$. As a consequence, the two-point function is expressed as

$$
\begin{aligned}
\left\langle A_{\mu}(x) A_{v}\left(x^{\prime}\right)\right\rangle_{0}= & -\frac{\eta_{\mu v} \alpha^{3-D}}{\pi^{(D-1) / 2}} \frac{\Gamma((D-1) / 2)}{z z^{\prime}\left(u^{2}-1\right)^{(D-1) / 2}}+\frac{\left(z z^{\prime}\right)^{D / 2-1}}{(2 \pi)^{D-2} \alpha^{D-3}} \\
& \times \int d \mathbf{k} \int_{0}^{\infty} d \lambda \frac{k_{\mu} k_{v}}{\lambda \omega} J_{D / 2-1}(\lambda z) J_{D / 2-1}\left(\lambda z^{\prime}\right) e^{i \mathbf{k} \cdot \Delta \mathbf{x}-i \omega \Delta t} .
\end{aligned}
$$

Though the second term in the right hand-side of this formula is not simplified, like the first one, the corresponding contribution to the two-point functions for the field tensor is further transformed to the form which is expressed in terms of the elementary functions (see below).

With the expression (3.8) we can evaluate the corresponding two-point functions for the field tensor $\left\langle 0\left|F_{\sigma \mu}(x) F_{\rho v}\left(x^{\prime}\right)\right| 0\right\rangle=\left\langle F_{\sigma \mu}(x) F_{\rho v}\left(x^{\prime}\right)\right\rangle_{0}$. First of all, we can see that the second term in the right-hand side of (3.8) does not contribute to the components $\left\langle F_{\sigma \mu}(x) F_{\rho v}\left(x^{\prime}\right)\right\rangle_{0}$ with $\sigma=$ $0,1, \ldots, D-1$. For these components we get

$$
\begin{aligned}
\left\langle F_{\sigma \mu}(x) F_{\rho v}\left(x^{\prime}\right)\right\rangle_{0} & =4 \frac{g_{[\mu v}(z) g_{\sigma] \rho}\left(z^{\prime}\right)+g_{\sigma[\rho}(z) g_{\mu v]}\left(z^{\prime}\right)}{\pi^{(D-1) / 2} \alpha^{D+1}} \frac{\Gamma((D+1) / 2) u}{\left(u^{2}-1\right)^{(D+1) / 2}}, \\
\left\langle F_{\sigma \mu}(x) F_{z v}\left(x^{\prime}\right)\right\rangle_{0} & =\frac{2 \Gamma((D-1) / 2)}{\pi^{(D-1) / 2} \alpha^{D-3}} \eta_{[\sigma v} \partial_{\mu]} \partial_{z}^{\prime} \frac{1}{z z^{\prime}\left(u^{2}-1\right)^{(D-1) / 2}},
\end{aligned}
$$


for $\sigma, \rho=0,1, \ldots, D-1$. Here the square brackets in the index expressions mean the antisymmetrization with respect to the indices enclosed. The last term in the right-hand side of (3.8) contributes only to the components $\left\langle F_{z \mu}(x) F_{z v}\left(x^{\prime}\right)\right\rangle_{0}$ and to the ones obtained from this by the interchange of the indices. The corresponding integral is evaluated in a way similar to that for (3.6):

$$
\int d \mathbf{k} \int_{0}^{\infty} d \lambda \frac{\lambda}{\omega} J_{D / 2-2}(\lambda z) J_{D / 2-2}\left(\lambda z^{\prime}\right) e^{i \mathbf{k} \cdot \Delta \mathbf{x}-i \omega \Delta t}=\frac{2^{D-2} \pi^{(D-3) / 2} \Gamma((D-1) / 2) u}{\left(z z^{\prime}\right)^{D / 2}\left(u^{2}-1\right)^{(D-1) / 2}} .
$$

For the two-point function this gives

$$
\left\langle F_{z \mu}(x) F_{z v}\left(x^{\prime}\right)\right\rangle_{0}=\frac{\Gamma((D-1) / 2)}{\alpha^{D-3} \pi^{(D-1) / 2}}\left(\partial_{\mu} \partial_{v}^{\prime} u-\eta_{\mu v} \partial_{z} \partial_{z}^{\prime}\right) \frac{1}{z z^{\prime}\left(u^{2}-1\right)^{(D-1) / 2}},
$$

with $\mu, v=0,1, \ldots, D-1$.

\section{Two-point functions in the geometry with a boundary}

Now we assume the presence of a boundary located at $z=z_{0}$ on which the field obeys the boundary condition

$$
n^{\mu} F_{\mu v_{1} \cdots v_{D-1}}^{*}=0
$$

where $n^{\mu}$ is the normal vector to the boundary, $F_{\mu v_{1} \cdots v_{D-1}}^{*}$ is the dual of the field tensor $F_{\mu v}$. For $D=3$ this corresponds to the perfectly conducting boundary condition. Here we consider the region $z_{0}<z<\infty$. The mode functions in this region are given by the expression (2.10) where $Z_{D / 2-1}(\lambda z)$ is a linear combination of the functions $J_{D / 2-1}(\lambda z)$ and $Y_{D / 2-1}(\lambda z)$, where $Y(x)$ is the Neumann function. The relative coefficient in this combination is determined from the boundary condition. By taking into account that in the gauge at hand $A_{(\sigma \lambda \mathbf{k}) D}=0$, from the boundary condition it follows that $\left.F_{\mu v}\right|_{z=0}=0$ for $\mu, v=0,1, \ldots, D-1$. From here for the mode functions we find

$$
A_{(\sigma \lambda \mathbf{k}) \mu}=C \varepsilon_{(\sigma) \mu} z^{D / 2-1} g_{D / 2-1}\left(\lambda z_{0}, \lambda z\right) e^{i \mathbf{k} \cdot \mathbf{x}-i \omega t}
$$

with $\mu, v=0,1, \ldots, D-1$, and

$$
g_{D / 2-1}(x, y)=J_{D / 2-1}(y) Y_{D / 2-1}(x)-J_{D / 2-1}(x) Y_{D / 2-1}(y) .
$$

From the normalization condition (2.15), with the integration over $\left[z_{0}, \infty\right)$, for the coefficient $C$ one gets

$$
C^{2}=\frac{\alpha^{3-D} \lambda}{(2 \pi)^{D-2} \omega}\left[J_{D / 2-1}^{2}\left(\lambda z_{0}\right)+Y_{D / 2-1}^{2}\left(\lambda z_{0}\right)\right]^{-1} .
$$

With the mode functions (4.2), for the two-point functions of the vector potential one finds

$$
\begin{aligned}
\left\langle A_{\mu}(x) A_{v}\left(x^{\prime}\right)\right\rangle= & \frac{\left(z z^{\prime}\right)^{D / 2-1}}{(2 \pi)^{D-2} \alpha^{D-3}} \int d \mathbf{k} \int_{0}^{\infty} d \lambda \frac{\lambda}{\omega}\left(-\eta_{\mu v}+\frac{k_{\mu} k_{v}}{\lambda^{2}}\right) \\
& \times \frac{g_{D / 2-1}\left(\lambda z_{0}, \lambda z\right) g_{D / 2-1}\left(\lambda z_{0}, \lambda z^{\prime}\right)}{Y_{D / 2-1}^{2}\left(\lambda z_{0}\right)+J_{D / 2-1}^{2}\left(\lambda z_{0}\right)} e^{i \mathbf{k} \cdot \Delta \mathbf{x}-i \omega \Delta t} .
\end{aligned}
$$


We are interested in effects induced by the boundary. In order to separate from the two-point function the boundary-induced contribution, we subtract from (4.5) the two-point function for the boundary-free geometry, given by (3.5). By using the relation

$$
\begin{aligned}
\frac{g_{D / 2-1}\left(\lambda z_{0}, \lambda z\right) g_{D / 2-1}\left(\lambda z_{0}, \lambda z^{\prime}\right)}{Y_{D / 2-1}^{2}\left(\lambda z_{0}\right)+J_{D / 2-1}^{2}\left(\lambda z_{0}\right)}= & J_{D / 2-1}(\lambda z) J_{D / 2-1}\left(\lambda z^{\prime}\right) \\
& -\frac{1}{2} \sum_{j=1,2} \frac{J_{D / 2-1}\left(\lambda z_{0}\right)}{H_{D / 2-1}^{(j)}\left(\lambda z_{0}\right)} H_{D / 2-1}^{(j)}(\lambda z) H_{D / 2-1}^{(j)}\left(\lambda z^{\prime}\right)
\end{aligned}
$$

with $H_{v}^{(j)}(x), j=1,2$, being the Hankel functions, the boundary-induced contribution is presented as

$$
\begin{aligned}
\left\langle A_{\mu}(x) A_{v}\left(x^{\prime}\right)\right\rangle_{b}= & \left\langle A_{\mu}(x) A_{v}\left(x^{\prime}\right)\right\rangle-\left\langle A_{\mu}(x) A_{v}\left(x^{\prime}\right)\right\rangle_{0} \\
= & -\frac{\left(z z^{\prime}\right)^{D / 2-1}}{2(2 \pi)^{D-2} \alpha^{D-3}} \int d \mathbf{k} e^{i \mathbf{k} \cdot \Delta \mathbf{x}} \sum_{j=1,2} \int_{0}^{\infty} d \lambda \frac{\lambda}{\omega} \frac{J_{D / 2-1}\left(\lambda z_{0}\right)}{H_{D / 2-1}^{(j)}\left(\lambda z_{0}\right)} \\
& \times H_{D / 2-1}^{(j)}(\lambda z) H_{D / 2-1}^{(j)}\left(\lambda z^{\prime}\right) e^{-i \omega \Delta t}\left(-\eta_{\mu v}+\frac{k_{\mu} k_{v}}{\lambda^{2}}\right)
\end{aligned}
$$

As the next step, under the condition $z+z^{\prime}>2 z_{0}+|\Delta t|$, we rotate the integration contour in the complex plane $\lambda$ by the angle $\pi / 2$ for the term with $j=1$ and by the angle $-\pi / 2$ for $j=2$. Introducing the modified Bessel functions, for the boundary-induced part of the two-point function we get the expression

$$
\begin{aligned}
\left\langle A_{\mu}(x) A_{v}\left(x^{\prime}\right)\right\rangle_{b}= & \frac{4\left(z z^{\prime}\right)^{D / 2-1}}{(2 \pi)^{D-1} \alpha^{D-3}} \int d \mathbf{k} \int_{k}^{\infty} d x\left(\eta_{\mu v} x^{2}+\partial_{\mu} \partial_{v}^{\prime}\right) e^{i \mathbf{k} \cdot \Delta \mathbf{x}} \\
& \times \frac{\cosh \left(\Delta t \sqrt{x^{2}-k^{2}}\right)}{x \sqrt{x^{2}-k^{2}}} f_{D / 2-1}\left(x z_{0}, x z, x z^{\prime}\right),
\end{aligned}
$$

with $\mu, v=0,1, \ldots, D-1$ and the other components vanish. In (4.8) we use the notation

$$
f_{D / 2-1}(u, v, w)=\frac{I_{D / 2-1}(u)}{K_{D / 2-1}(u)} K_{D / 2-1}(v) K_{D / 2-1}(w),
$$

where $I_{v}(x)$ and $K_{V}(x)$ are the modified Bessel functions.

Having the boundary-induced contribution to the two-point function for the vector potential we can find the corresponding contribution to the two-point function for the field tensor by differentiations. First of all for the components with $\mu, v, \rho, \sigma=1, \ldots, D-1$ we get

$$
\begin{aligned}
\left\langle F_{\sigma \mu}(x) F_{\rho v}\left(x^{\prime}\right)\right\rangle_{b}= & -\frac{8\left(z z^{\prime}\right)^{D / 2-1}}{(2 \pi)^{D-1} \alpha^{D-3}} \int d \mathbf{k}\left(\delta_{[\mu v} k_{\sigma]} k_{\rho}+\delta_{\sigma[\rho} k_{\mu} k_{v]}\right) e^{i \mathbf{k} \cdot \Delta \mathbf{x}} \\
& \times \int_{k}^{\infty} d x \frac{x \cosh \left(\Delta t \sqrt{x^{2}-k^{2}}\right)}{\sqrt{x^{2}-k^{2}}} f_{D / 2-1}\left(x z_{0}, x z, x z^{\prime}\right) .
\end{aligned}
$$


The components with one index being zero are given by

$$
\begin{aligned}
\left\langle F_{0 \mu}(x) F_{\rho v}\left(x^{\prime}\right)\right\rangle_{b}= & \left\langle F_{\rho v}(x) F_{0 \mu}\left(x^{\prime}\right)\right\rangle_{b} \\
= & -\frac{8 i\left(z z^{\prime}\right)^{D / 2-1}}{(2 \pi)^{D-1} \alpha^{D-3}} \int d \mathbf{k} \delta_{\mu[v} k_{\rho]} e^{i \mathbf{k} \cdot \Delta \mathbf{x}} \int_{k}^{\infty} d x \\
& \times x \sinh \left(\Delta t \sqrt{x^{2}-k^{2}}\right) f_{D / 2-1}\left(x z_{0}, x z, x z^{\prime}\right),
\end{aligned}
$$

where $\mu, v, \rho=1, \ldots, D-1$. Next, for the components with two indices being zero one has

$$
\begin{aligned}
\left\langle F_{0 \mu}(x) F_{0 v}\left(x^{\prime}\right)\right\rangle_{b}= & \frac{4\left(z z^{\prime}\right)^{D / 2-1}}{(2 \pi)^{D-1} \alpha^{D-3}} \int d \mathbf{k} e^{i \mathbf{k} \cdot \Delta \mathbf{x}} \int_{k}^{\infty} d x x\left[\left(x^{2}-k^{2}\right) \delta_{\mu v}+k_{\mu} k_{v}\right] \\
& \times \frac{\cosh \left(\Delta t \sqrt{x^{2}-k^{2}}\right)}{\sqrt{x^{2}-k^{2}}} f_{D / 2-1}\left(x z_{0}, x z, x z^{\prime}\right),
\end{aligned}
$$

with $\mu, v=1, \ldots, D-1$.

Now let us consider the functions having $D$-components. After the appropriate differentiations we get $(\mu, v=1, \ldots, D-1)$

$$
\begin{aligned}
\left\langle F_{D \mu}(x) F_{\rho v}\left(x^{\prime}\right)\right\rangle_{b}= & -\frac{8 i z^{D / 2-1}}{(2 \pi)^{D-1} \alpha^{D-3}} \int d \mathbf{k} \delta_{\mu[v} k_{\rho]} e^{i \mathbf{k} \cdot \Delta \mathbf{x}} \int_{k}^{\infty} d x x \\
& \times \frac{\cosh \left(\Delta t \sqrt{x^{2}-k^{2}}\right)}{\sqrt{x^{2}-k^{2}}} \partial_{z}\left[z^{D / 2-1} f_{D / 2-1}\left(x z_{0}, x z, x z^{\prime}\right)\right], \\
\left\langle F_{D \mu}(x) F_{D v}\left(x^{\prime}\right)\right\rangle_{b}= & \frac{4 \alpha^{3-D}}{(2 \pi)^{D-1}} \int d \mathbf{k} e^{i \mathbf{k} \cdot \Delta \mathbf{x}} \int_{k}^{\infty} d x x \frac{\cosh \left(\Delta t \sqrt{x^{2}-k^{2}}\right)}{\sqrt{x^{2}-k^{2}}} \\
& \times \partial_{z} \partial_{z^{\prime}}\left[\left(z z^{\prime}\right)^{D / 2-1} f_{D / 2-1}\left(x z_{0}, x z, x z^{\prime}\right)\right]\left(\frac{k_{\mu} k_{v}}{x^{2}}-\delta_{\mu v}\right),
\end{aligned}
$$

and

$$
\begin{aligned}
\left\langle F_{0 D}(x) F_{0 D}\left(x^{\prime}\right)\right\rangle_{b}= & \frac{4 \alpha^{3-D}}{(2 \pi)^{D-1}} \int d \mathbf{k} e^{i \mathbf{k} \cdot \Delta \mathbf{x}} \int_{k}^{\infty} d x \frac{\cosh \left(\Delta t \sqrt{x^{2}-k^{2}}\right)}{\sqrt{x^{2}-k^{2}}} \\
& \partial_{z} \partial_{z^{\prime}}\left[\left(z z^{\prime}\right)^{D / 2-1} f_{D / 2-1}\left(x z_{0}, x z, x z^{\prime}\right)\right] \frac{k^{2}}{x}
\end{aligned}
$$

All the remaining components are zero. In the evaluation of the VEVs of local observables bilinear in the field we need to have the coincidence limit of the boundary-induced parts in the two-point functions. For points away from the boundary, this limit is finite. All the divergences are contained in the boundary-free part. For the illustration of the details in the evaluation of the coincidence limit we consider the components

$$
\begin{aligned}
\left\langle F_{\sigma \mu}(x) F_{\rho v}(x)\right\rangle_{b}= & -\frac{4 z^{D-2}\left(\delta_{\mu v} \delta_{\sigma \rho}-\delta_{\mu \rho} \delta_{\sigma v}\right)}{(2 \pi)^{D-1} \alpha^{D-3}} \int d \mathbf{k}\left(k_{\mu}^{2}+k_{\sigma}^{2}\right) \\
& \times \int_{k}^{\infty} d u \frac{u f_{D / 2-1}\left(u z_{0}, u z, u z\right)}{\sqrt{u^{2}-k^{2}}}
\end{aligned}
$$


with $\sigma, \mu, \rho, v=1, \ldots, D-1$. By taking into account that

$$
\int d \mathbf{k} k_{\mu}^{2} g(k)=\frac{\pi^{(D-1) / 2}}{\Gamma((D+1) / 2)} \int_{0}^{\infty} d k k^{D} g(k),
$$

this functions is written as

$$
\begin{aligned}
\left\langle F_{\sigma \mu}(x) F_{\rho \nu}(x)\right\rangle_{b}= & -\frac{8 z^{D-2}\left(\delta_{\mu \nu} \delta_{\sigma \rho}-\delta_{\mu \rho} \delta_{\sigma \nu}\right)}{(4 \pi)^{(D-1) / 2} \alpha^{D-3} \Gamma((D+1) / 2)} \\
& \times\left.\int_{0}^{\infty} d k k^{D} \int_{0}^{\infty} d y f_{D / 2-1}\left(u z_{0}, u z, u z\right)\right|_{u=\sqrt{k^{2}+y^{2}}}
\end{aligned}
$$

Introducing polar coordinates in the $(k, y)$-plane, after the integration of the angular part we get the final expression. Other components are evaluated in a similar way and one finds

$$
\begin{aligned}
\left\langle F_{\sigma \mu}(x) F_{\rho v}(x)\right\rangle_{b} & =-\frac{2\left(\eta_{\mu v} \eta_{\sigma \rho}-\eta_{\mu \rho} \eta_{\sigma v}\right) z^{D-2}}{(4 \pi)^{D / 2-1} \Gamma(D / 2+1) \alpha^{D-3}} \int_{0}^{\infty} d u u^{D+1} f_{D / 2-1}\left(u z_{0}, u z, u z\right), \\
\left\langle F_{D \mu}(x) F_{D v}(x)\right\rangle_{b} & =\frac{\eta_{\mu v}(D-1) \alpha^{3-D} z^{D-2}}{(4 \pi)^{D / 2-1} \Gamma(D / 2+1)} \int_{0}^{\infty} d u u^{D+1} \frac{I_{D / 2-1}\left(u z_{0}\right)}{K_{D / 2-1}\left(u z_{0}\right)} K_{D / 2-2}^{2}(u z),
\end{aligned}
$$

for $\sigma, \mu, \rho, v=0,1, \ldots, D-1$. Remaining components vanish.

The consideration for the region $0<z<z_{0}$ is done in a similar way. The corresponding mode functions, regular on the AdS boundary, are obtained from the expression (4.2) by the replacement $g_{D / 2-1}\left(\lambda z_{0}, \lambda z\right) \rightarrow J(\lambda z)$. From the boundary condition on the plate $z=z_{0}$ it follows that the eigenvalues for $\lambda$ are roots of the equation $J_{D / 2-1}\left(\lambda z_{0}\right)=0$. The mode-sum for the two-point function contains series over these eigenvalues. For the summation of the series we use the generalized Abel-Plana formula from [9]. As a result the two-point function is presented in the decomposed form (the details will be presented elsewhere)

$$
\left\langle A_{\mu}(x) A_{v}\left(x^{\prime}\right)\right\rangle=\left\langle A_{\mu}(x) A_{v}\left(x^{\prime}\right)\right\rangle_{0}+\left\langle A_{\mu}(x) A_{v}\left(x^{\prime}\right)\right\rangle_{b},
$$

where the boundary-induced contribution is given by

$$
\begin{aligned}
\left\langle A_{\mu}(x) A_{v}\left(x^{\prime}\right)\right\rangle_{b}= & \frac{4\left(z z^{\prime}\right)^{D / 2-1}}{(2 \pi)^{D-1} \alpha^{D-3}} \int d \mathbf{k} \int_{k}^{\infty} d x\left(\eta_{\mu v} x^{2}+\partial_{\mu} \partial_{v}^{\prime}\right) e^{i \mathbf{k} \cdot \Delta \mathbf{x}} \\
& \times \frac{\cosh \left(\Delta t \sqrt{x^{2}-k^{2}}\right)}{x \sqrt{x^{2}-k^{2}}} \frac{K_{D / 2-1}\left(x z_{0}\right)}{I_{D / 2-1}\left(x z_{0}\right)} I_{D / 2-1}(x z) I_{D / 2-1}\left(x z^{\prime}\right) .
\end{aligned}
$$

The latter is related to the corresponding expression in the region $z_{0}<z<\infty$ by the replacements $I_{v} \rightleftarrows K_{v}$. The corresponding formulas for the two-point functions of the field tensor are obtained from those given above by making these replacements.

\section{VEV of the energy-momentum tensor}

It is well known that the important characteristic of the vacuum state is the VEV of the energymomentum tensor. It acts as the source of gravity in the quasiclassical Einstein equations and plays 
an important role in modelling self-consistent dynamics involving the gravitational field. The VEV of the energy-momentum tensor is decomposed into the boundary-free and boundary-induced parts:

$$
\left\langle T_{v}^{\mu}\right\rangle=\left\langle T_{v}^{\mu}\right\rangle_{0}+\left\langle T_{v}^{\mu}\right\rangle_{b}
$$

For points outside the plate the renormalization is required for the boundary-free part only. Because of the maximal symmetry of the background geometry, the latter is proportional to the metric tensor: $\left\langle T_{v}^{\mu}\right\rangle_{0}=$ const $\cdot \delta_{v}^{\mu}$. Here we are interested in the boundary-induced part which is directly evaluated with the help of the formula

$$
\left\langle T_{v}^{\mu}\right\rangle_{b}=-\frac{1}{4 \pi}\left\langle F_{\mu \beta}(x) F^{v \beta}(x)\right\rangle_{b}+\frac{\delta_{v}^{\mu}}{16 \pi}\left\langle F_{\beta \sigma}(x) F^{\beta \sigma}(x)\right\rangle_{b} .
$$

By using the expressions for the two-point functions in the coincidence limit, we can see that the vacuum energy-momentum tensor is diagonal. For the boundary-induced contributions in the VEVs of the diagonal components one gets (no summation over $l$ )

$$
\left\langle T_{l}^{l}\right\rangle_{b}=-\frac{(D-1)\left(z / z_{0}\right)^{D+2}}{(4 \pi)^{D / 2} \Gamma(D / 2+1) \alpha^{D+1}} \int_{0}^{\infty} d x x^{D+1} \frac{I_{D / 2-1}(x)}{K_{D / 2-1}(x)} G_{D / 2-1}^{(l)}\left(x z / z_{0}\right),
$$

where

$$
\begin{aligned}
G_{v}^{(l)}(u) & =v K_{v-1}^{2}(u)+(v-1) K_{v}^{2}(u), l=0,1, \ldots, D-1, \\
G_{v}^{(D)}(u) & =(v+1)\left[K_{v}^{2}(u)-K_{v-1}^{2}(u)\right] .
\end{aligned}
$$

It can be seen that $G_{1 / 2}^{(l)}(u)=0$ and, hence, the boundary-induced contribution vanishes for $D=3$. Of course, we could expect this result from the conformal relation to the corresponding problem in Minkowski bulk. From (5.4), by taking into account that $K_{v}(u)>K_{v-1}(u)$ for $v \geqslant 1$, we conclude that for $D \geqslant 4$ all the components of the boundary-induced VEV are negative: $\left\langle 0\left|T_{l}^{l}\right| 0\right\rangle_{b}<0$. The boundary-induced contribution in the VEV of the energy-momentum tensor for the region $0<z<z_{0}$ is obtained from (5.3) and (5.4) by the replacements $I_{V} \rightleftarrows K_{V}$.

Note that the boundary-induced VEVs (5.3) depend on $z$ and $z_{0}$ in the form of the ratio $z / z_{0}$ which is related to the proper distance from the boundary, $y-y_{0}$, by $z / z_{0}=e^{\left(y-y_{0}\right) / \alpha}$. Hence, for a given proper distance from the boundary, the VEVs do not depend on the location of the boundary. The latter property is a consequence of the maximal symmetry of the AdS spacetime.

It can be seen that the boundary-induced contributions in the VEV of the energy-momentum tensor obey the continuity equation $\nabla_{k}\left\langle T_{i}^{k}\right\rangle_{b}=0$ which for the geometry under consideration takes the form

$$
z^{D+1} \partial_{z}\left(z^{-D}\left\langle T_{D}^{D}\right\rangle_{b}\right)+D\left\langle T_{0}^{0}\right\rangle_{b}=0
$$

Let us consider the asymptotic behavior of the VEVs at large distances from the plate and near the plate. At distances larger that the AdS curvature radius one has $y-y_{0} \gg \alpha$ and, hence, $z / z_{0} \gg 1$. In (5.3) we introduce a new integration variable $u=x z / z_{0}$ and use the asymptotic expressions for the modified Bessel functions for small arguments. For the VEV of the energy-momentum tensor we find

$$
\left\langle T_{D}^{D}\right\rangle_{b} \approx \frac{D\left\langle T_{0}^{0}\right\rangle_{b}}{2(D-1)}
$$


and

$$
\left\langle T_{0}^{0}\right\rangle_{b} \approx-\frac{(D-1)^{2}(D-2)(D-3)}{2 \pi^{D / 2} \alpha^{D+1}\left(z / z_{0}\right)^{D-2}} \frac{\Gamma^{2}(D) \Gamma(3 D / 2-2)}{\Gamma^{2}(D / 2) \Gamma(2 D)} .
$$

Hence, at large distances the VEVs are suppressed as functions of the proper distance by the factor $e^{-(D-2)\left(y-y_{0}\right) / \alpha}$.

At small distances from the plate, compared with the AdS curvature radius, one has $y-y_{0} \ll \alpha$ and, hence, $1-z_{0} / z \ll 1$. In this case the dominant contribution to the integrals in (5.3) comes from the region with $x \sim 1 /\left(z / z_{0}-1\right) \gg 1$ we can use the asymptotic expressions for the modified Bessel functions for large values of the argument. To the leading order this gives

$$
\begin{aligned}
\left\langle T_{0}^{0}\right\rangle_{b} & \approx-\frac{(D-1)(D-3) \Gamma((D+1) / 2)}{2(4 \pi)^{(D+1) / 2}\left(y-y_{0}\right)^{D+1}}, \\
\left\langle T_{D}^{D}\right\rangle_{b} & \approx-\frac{(D-1)(D-3) \Gamma((D+1) / 2)}{2(4 \pi)^{(D+1) / 2} \alpha\left(y-y_{0}\right)^{D}},
\end{aligned}
$$

for the components of the energy-momentum tensor. The leading terms for the VEVs of the energy density do not depend on the curvature radius $\alpha$ and coincide with the corresponding results for the plate in Minkowski spacetime. Hence, for these VEVs near the plate the effects of gravity are small.

\section{Conclusion}

We have discussed the effects of a boundary in AdS spacetime on the properties of the electromagnetic vacuum in an arbitrary number of spatial dimensions. The boundary is parallel to the AdS boundary and on it the electromagnetic field obeys the condition (4.1). For $D=3$ the latter corresponds to the standard perfect conductor boundary condition. First we have considered the complete set of mode functions for the electromagnetic field in the boundary-free AdS spacetime, given by (3.3). With these functions, the two-point functions for the vector potential is expressed as (3.8). Though the last term in this formula is not expressed in terms of elementary functions, its contribution to the two-point functions for the field tensor is simplified and one gets simple expressions (3.9) and (3.11).

In the region $y>y_{0}$, the mode functions obeying the boundary condition (4.1) at $y=y_{0}$, are given by (4.2) and for the two-point function of the vector potential one gets the expression (4.5). In order to extract the part induced by the boundary, we subtracted from the two-point function the corresponding function in the boundary-free AdS spacetime. After the appropriate rotations of the integration contours, the boundary-induced part in the two-point function for the vector potential is written in the form (4.8). The corresponding part in the two-point function of the field tensor is obtained by simple differentiations. In particular, for the evaluation of the VEVs for physical quantities bilinear in the field, we need the boundary-induced part of the two-point function in the coincidence limit of the arguments. For points away from the boundary the latter is finite and the nonzero components are given by the expressions (4.19). The divergences are contained in the boundary-free parts only and the renormalization is reduced to the one for the boundary-free geometry. The expressions of the boundary-induced contributions in the two-point functions for the region $y<y_{0}$ are obtained from those in the region $y>y_{0}$ by the replacements $I_{v} \rightleftarrows K_{v}$.. 
In section 5 we have investigated the boundary-induced contributions in the VEV of the energy-momentum tensor. For spatial dimensions $D \geqslant 4$, the boundary-induced contributions to all the components of the energy-momentum tensor are negative. At distances from the boundary much larger than the AdS curvature radius the boundary-induced contributions are suppressed by the factor $e^{-(D-2)\left(y-y_{0}\right) / \alpha}$. For points near the boundary the contribution of the vacuum fluctuations with the wavelengths much smaller that the AdS curvature radius dominates and the boundary-induced VEVs, in the leading order, coincides with the corresponding VEVs for a plate in Minkowski bulk. We have shown that the boundary-induced energy density is negative and the normal stress corresponds to positive pressure. The results obtained can be used for the investigation of the vacuum characteristics in the geometry of two parallel plates, including the Casimir forces acting on the plates.

\section{References}

[1] V.M. Mostepanenko, N.N. Trunov, The Casimir Effect and Its Applications (Oxford University Press, Oxford, 1997); K.A. Milton, The Casimir Effect: Physical Manifestation of Zero-Point Energy (World Scientific, Singapore, 2002); M. Bordag, G.L. Klimchitskaya, U. Mohideen, V.M. Mostepanenko, Advances in the Casimir Effect (Oxford University Press, Oxford, 2009); Casimir Physics, edited by D. Dalvit, P. Milonni, D. Roberts, F. da Rosa, Lecture Notes in Physics Vol. 834 (Springer-Verlag, Berlin, 2011).

[2] W. Goldberger, I. Rothstein, Phys. Lett. B 491, 339 (2000); S. Nojiri, S.D. Odintsov, S. Zerbini, Class. Quantum Grav. 17, 4855 (2000); A. Flachi, I.G. Moss, D.J. Toms, Phys. Lett. B 518, 153 (2001); I. Brevik, K.A. Milton, S. Nojiri, S.D. Odintsov, Nucl. Phys. B 599, 305 (2001); A. Flachi, I.G. Moss, D.J. Toms, Phys. Rev. D 64, 105029 (2001).J. Garriga, O. Pujolàs, T. Tanaka, Nucl. Phys. B 605, 192 (2001); A. Flachi, D.J. Toms, Nucl. Phys. B 610, 144 (2001); J. Garriga, A. Pomarol, Phys. Lett. B 560, 91 (2003); E. Elizalde, S. Nojiri, S.D. Odintsov, S. Ogushi, Phys. Rev. D 67, 063515 (2003); A.A. Saharian, M.R. Setare, Phys. Lett. B 584, 306 (2004); A. Flachi, A. Knapman, W. Naylor, M. Sasaki, Phys. Rev. D 70, 124011 (2004); E. Elizalde, S. Nojiri, S.D. Odintsov, Phys. Rev. D 70, 043539 (2004); E. Elizalde, S. Nojiri, S.D. Odintsov, P. Wang, Phys. Rev. D 71, 103504 (2005); M. Frank, I. Turan, L. Ziegler, Phys. Rev. D 76, 015008 (2007); L.P. Teo, Phys. Lett. B 682, 259 (2009); A. Flachi, T. Tanaka, Phys. Rev. D 80, 124022 (2009); M. Rypestøl, I. Brevik, New J. Phys 12, 013022 (2010).

[3] A. Knapman, D.J. Toms, Phys. Rev. D 69, 044023 (2004); A.A. Saharian, Nucl. Phys. B 712, 196 (2005); A.A. Saharian, Phys. Rev. D 70, 064026 (2004); S.-H. Shao, P. Chen, J.-A. Gu, Phys. Rev. D 81, 084036 (2010); E. Elizalde, S.D. Odintsov, A.A. Saharian, Phys. Rev. D 87, 084003 (2013).

[4] A. Flachi, J. Garriga, O. Pujolàs, T. Tanaka, J. High Energy Phys. 08 (2003) 053; A. Flachi, O. Pujolàs, Phys. Rev. D 68, 025023 (2003); A.A. Saharian, Phys. Rev. D 73, 044012 (2006); A.A. Saharian, Phys. Rev. D 73, 064019 (2006); A.A. Saharian, Phys. Rev. D 74, 124009 (2006); E. Elizalde, M. Minamitsuji, W. Naylor, Phys. Rev. D 75, 064032 (2007); R. Linares, H.A. Morales-Técotl, O. Pedraza, Phys. Rev. D 77, 066012 (2008); M. Frank, N. Saad, I. Turan, Phys. Rev. D 78, 055014 (2008); E. Elizalde, S.D. Odintsov, A.A. Saharian, Phys. Rev. D 79, 065023 (2009).

[5] E.R. Bezerra de Mello, A.A. Saharian, V. Vardanyan, Phys. Lett. B 741, 155 (2015); S. Bellucci, A.A. Saharian, V. Vardanyan, JHEP 11(2015)092; S. Bellucci, A.A. Saharian, V. Vardanyan, arXiv: 1512.06569 .

[6] B. Allen, T. Jacobson, Commun. Math. Phys. 103, 699 (1986). 
[7] A.A Saharian, A.S. Kotanjyan, H.A. Nersisyan, Phys. Lett. B 728, 141 (2014); A.S Kotanjyan, A.A Saharian, H.A Nersisyan, Phys. Scr. 90, 065304 (2015); A.S. Kotanjyan, A.A. Saharian, H.A. Nersisyan, J. Phys.: Conf. Ser. 496, 012027 (2014).

[8] S. Bellucci, A.A. Saharian, Phys. Rev. D 88, 064034 (2013).

[9] A.A. Saharian, The Generalized Abel-Plana Formula with Applications to Bessel Functions and Casimir Effect (Yerevan State University Publishing House, Yerevan, 2008); Report No. ICTP/2007/082; arXiv:0708.1187. 